\title{
Incidence and Risk Factors of Recipient Surgical Site Infection in Oral and Maxillofacial Reconstruction with Vascularized Fibular Bone Grafts
}

\author{
Pichai Vittayakittipong, D.D.S., M.D. ${ }^{1}$, Sarocha Makmanee, D.D.S. ${ }^{2}$ \\ 'Department of Oral and Maxillofacial Surgery, Faculty of Dentistry, Prince of Songkla University, Hat Yai, Songkhla 90110, \\ Thailand. \\ ²Dental Unit, Sathing Phra Hospital, Sathing Phra, Songkhla 90190, Thailand. \\ Received 2 December 2019 • Revised 30 March 2020 • Accepted 1 April 2020 • Published online 15 May 2020
}

\begin{abstract}
:
Objective: To determine the incidence and risk factors of recipient surgical site infection (RSSI) after oral and maxillofacial reconstruction with vascularized fibular bone grafts (VFBG).

Material and Methods: This retrospective study was performed in patients who underwent oral and maxillofacial reconstructive surgery, with VFBG, at the Dental Hospital, Faculty of Dentistry, Prince of Songkla University. Demographic, preoperative, intraoperative and postoperative data were recorded. Any infection at the recipient site occurring within 30 days post-operatively, by criteria from the Center of Disease Control, was defined as RSSI. Statistical analysis was performed by chi-square test, Student's t-test and Pearson's correlation coefficient with statistical significance was set at 0.05 .
\end{abstract}

Results: There were twenty-one patients who met the eligibility criteria. The incidence of RSSI after oral and maxillofacial reconstruction with VFBG was $47.6 \%$ (10/21 patients), and the success rate of VFBG was $95.2 \%$. American Society of Anesthetics (ASA) physical status class II and oral contamination were significant risk factors for RSSI $(p-v a l u e=0.004$ and $p$-value $=0.031$, respectively). Length of hospital stay was significantly higher in the RSSI group ( $p-v a l u e<0.001)$. Conclusion: The incidence of RSSI after oral and maxillofacial reconstruction with VFBG was high, and ASA physical status class II and oral contamination were significant risk factors for RSSI.

Keywords: risk factor, surgical site infection, vascularized fibular bone graft

Contact: Assoc. Prof. Pichai Vittayakittipong, DDS., M.D., F.R.C.D.T. (OMFS), F.R.C.O.T. (FPRS) Department of Oral and Maxillofacial Surgery, Faculty of Dentistry, Prince of Songkla University, Hat Yai, Songkhla 90110, Thailand.

E-mail: pichai.v@hotmail.com

This is an open access article under the CC BY-NC-ND license

(http://www.jhsmr.org/index.php/jhsmr/about/editorialPolicies\#openAccessPolicy).

J Health Sci Med Res 2020;38(3):231-238 doi: $10.31584 / j h s m r .2020742$ www.jhsmr.org 


\section{Introduction}

Vascularized fibular bone grafts (VFBG) are the gold standard for mandibular reconstruction, because they have several advantages over other grafts, including potential reconstruction of long span defects, their bicortical properties; which can assist in placement of dental implants, the availability of muscular cuff and good quality skin paddle for soft tissue reconstruction, long and large vascular pedicle, two team approach and low donor site morbidity. ${ }^{1-3}$ However, microvascular reconstruction with VFBG is a major operation; operative time and blood loss are increased, which in turn results in a greater risk of postoperative complications. Surgical site infection (SSI), especially at the recipient site, is a common complication after head and neck cancer surgery with microvascular free flap reconstruction. ${ }^{4,5}$ The adverse outcomes of SSI include prolonged hospitalization, delayed wound healing, increased risk of free flap failure and delayed postoperative treatment with chemotherapy and radiotherapy. ${ }^{5,6}$ There are several risk factors for SSI in head and neck surgery, such as comorbidities, smoking and alcohol consumption, poor oral hygiene, American Society of Anesthetics (ASA) physical status (PS) classification, and body mass index (BMI). ${ }^{4,5,7-10}$ Therefore, oral and maxillofacial surgeons need to understand the incidence and risk factors of SSI, for prevention and improvement of treatment outcomes. In so saying, the incidence and risk factors of SSI after head and neck surgery varies among studies, and included both recipient and donor sites. ${ }^{4,5,7-9,11}$ Additionally, most of the previous SSI studies focused on the overview of head and neck cancer and reconstructive surgery; rather than specific oral and maxillofacial regions. No previous studies have investigated SSI after performing a single osteomyocutaneous free flap, especially the VFBG in oral and maxillofacial regions. The aims of this study were to determine the incidence and risk factors for SSI, focusing on recipient site after oral and maxillofacial reconstruction with vascularized fibular bone grafts.

\section{Material and Methods}

This retrospective study included patients whom underwent oral and maxillofacial reconstruction with VFBG, from 2009 to 2017, at the Dental Hospital, Faculty of Dentistry, Prince of Songkla University. The study was approved by the research ethics committee, Faculty of Dentistry, Prince of Songkla University. Inclusion criteria were patients who underwent VFBG for oral and maxillofacial reconstruction by the same surgeon during this period, having complete medical records. Data collection was performed by abstracting information from inpatient medical records, including (1) demographic characteristics; (2) preoperative data including oral hygiene status, systemic disease, ASA PS classification, heavy smoking status ( $\geq 25$ cigarettes per day) ${ }^{12}$, heavy alcohol consumption (for women, $\geq 8$ drinks per week ; for men, $\geq 15$ drinks per week) ${ }^{13}$, prior treatment with radiotherapy and chemotherapy, perioperative steroid use, drug abuse, BMI, timing of reconstruction, cause(s) of defect, Boyd's classification of mandibular defect ${ }^{14}$, Brown's classification of maxillary defect $^{15}$, and use of prophylactic antibiotics; (3) intraoperative data; including oral contamination (any intraoral wound that had contact with any extraoral surgical wound), volume of blood loss, blood transfusion, size of skin paddle, operation time, number of fibula segments used, length of defect and type of drain; (4) postoperative data, including length of hospital stay and final results of VFBG. Any infection at the recipient site occurring within 30 days postoperatively was classified as recipient SSI (RSSI), which was categorized as superficial incisional, deep incisional and organ/space by criteria for diagnosis of surgical site infection by the United States Center of Disease Control. ${ }^{16}$ Additional data recorded for all patients included: the date, type, and severity of infection, treatment (empirical anti- 
biotics, surgical intervention), gram stain, culture and sensitivity test. Data were presented descriptively, using means and standard deviations. Chi-square test was used for comparing categorical data and Student's t-test was used for comparing continuous data. The relationship between continuous variables was tested with Pearson's correlation coefficient and statistical significance was set at a $p$-value $<0.05$.

\section{Results}

Twenty-one patients were included in the study, eleven males $(52.4 \%)$ and ten females (47.6\%) with a mean age of $37.9 \pm 16.4$ years (range 15-65 years). The mean $\mathrm{BMI}$ was $24.8 \pm 6.0$ kilograms per square meter $\left(\mathrm{kg} / \mathrm{m}^{2}\right)$, with a range of $16.4-39.4 \mathrm{~kg} / \mathrm{m}^{2}$. Seven patients had systemic diseases, with hypertension being the most common (20.8\%), followed by diabetes mellitus, chronic hepatitis $\mathrm{C}$, hyperthyroidism and psychotic depression. All primary diseases had a benign pathology. Ameloblastoma was the most common pathology of the jaws (66.7\%), while the others were osteoradionecrosis (14.3\%), ossifying fibroma (9.5\%) and gunshot wound (9.5\%). Distributions of preoperative, intraoperative and postoperative data are shown in Tables 1 and 2. Mandibular reconstruction with VFBG was performed in 18 cases (85.5\%), while maxillary reconstruction was performed in 3 cases $(4.8 \%)$. Table 3 shows the classification of maxillary and mandibular defects.

Table 1 Relationship between factors and recipient surgical site infection (categorical data)

\begin{tabular}{|c|c|c|c|c|}
\hline Factor & Category & $\begin{array}{l}\text { Number of patients }(\%) \\
(n=21)\end{array}$ & RSSI & $p$-value \\
\hline \multirow[t]{2}{*}{ Gender } & Male & $10(47.6)$ & 4 & 0.505 \\
\hline & Female & $11(52.4)$ & 6 & \\
\hline \multirow[t]{2}{*}{ Systemic diseases } & Yes & 7 (33.3) & 4 & 0.537 \\
\hline & No & $14(66.7)$ & 6 & \\
\hline \multirow[t]{2}{*}{ ASA PS } & Class I & $9(42.9)$ & 2 & 0.044 \\
\hline & Class II & $12(57.1)$ & 8 & \\
\hline \multirow[t]{2}{*}{ Heavy smoking } & Yes & $3(14.3)$ & 2 & 0.476 \\
\hline & No & $18(85.7)$ & 8 & \\
\hline \multirow[t]{2}{*}{ Heavy alcohol consumption } & Yes & $6(28.6)$ & 4 & 0.269 \\
\hline & No & $15(71.4)$ & 6 & \\
\hline \multirow[t]{2}{*}{ Radiotherapy } & Yes & $3(14.3)$ & 3 & 0.050 \\
\hline & No & $18(85.7)$ & 7 & \\
\hline \multirow[t]{2}{*}{ Chemotherapy } & Yes & $1(4.8)$ & 1 & NA \\
\hline & No & $20(95.2)$ & 9 & \\
\hline \multirow[t]{2}{*}{ Steroid use } & Yes & $14(66.7)$ & 8 & 0.217 \\
\hline & No & 7 (33.3) & 2 & \\
\hline \multirow[t]{2}{*}{ Oral hygiene } & Fair & $20(95.2)$ & 10 & 0.329 \\
\hline & Poor & $1(4.8)$ & 0 & \\
\hline \multirow[t]{2}{*}{ BMI $\left(\mathrm{kg} / \mathrm{m}^{2}\right)$} & $<23$ & $10(47.6)$ & 3 & 0.123 \\
\hline & $\geq 23$ & $11(52.4)$ & 7 & \\
\hline \multirow[t]{2}{*}{ Timing of reconstruction } & Immediate & $11(52.4)$ & 7 & 0.123 \\
\hline & Delayed & $10(47.6)$ & 3 & \\
\hline \multirow[t]{2}{*}{ Oral contamination } & Yes & $14(66.7)$ & 9 & 0.031 \\
\hline & No & 7 (33.3) & 1 & \\
\hline
\end{tabular}


Table 1 (continued)

\begin{tabular}{lllll}
\hline Factor & Category & $\begin{array}{l}\text { Number of patients }(\%) \\
(\mathbf{n = 2 1 )}\end{array}$ & RSSI & p-value \\
\hline \multirow{2}{*}{ Blood transfusion } & Yes & $11(52.4)$ & 6 & 0.505 \\
Type of drain & No & $10(47.6)$ & 4 & 0.234 \\
Fibular osteotomy & Penrose & $17(81.0)$ & 3 & 0.593 \\
& Vacuum & $4(19.0)$ & 9 & 1 \\
\hline
\end{tabular}

RSSI=recipient surgical site infection, ASA PS=American Society of Anesthetics physical status, BMl=body mass index, $\mathrm{kg} / \mathrm{m}^{2}=\mathrm{kilogram}$ per square meter, $\mathrm{NA}=$ not available

Table 2 Relationship between factors and recipient surgical site infection (continuous data)

\begin{tabular}{|c|c|c|c|c|}
\hline \multirow{2}{*}{ Factor } & \multicolumn{3}{|c|}{ Mean士S.D. } & \multirow{2}{*}{$p$-value } \\
\hline & Overall $(n=21)$ & No RSSI $(n=11)$ & RSSI $(n=10)$ & \\
\hline Age (yr) & $37.9 \pm 16.4$ & $34.4 \pm 17.4$ & $41.7 \pm 15.1$ & 0.319 \\
\hline BMI $\left(\mathrm{kg} / \mathrm{m}^{2}\right)$ & $24.8 \pm 6.0$ & $23.6 \pm 5.8$ & $26.0 \pm 6.2$ & 0.385 \\
\hline Length of defect $(\mathrm{cm})$ & $9.9 \pm 3.2$ & $9.7 \pm 3.3$ & $10.2 \pm 3.1$ & 0.717 \\
\hline No. of fibular segment & $2.3 \pm 0.8$ & $2.2 \pm 0.8$ & $2.5 \pm 0.9$ & 0.374 \\
\hline Skin paddle size $\left(\mathrm{cm}^{2}\right)$ & $24.1 \pm 15.4$ & $28.8 \pm 20.6$ & $20.6 \pm 9.8$ & 0.307 \\
\hline Operation time (min) & $800.0 \pm 122.3$ & $770.9 \pm 131.3$ & $832.0 \pm 109.0$ & 0.263 \\
\hline Blood loss (ml) & $778.6 \pm 165.5$ & $781.8 \pm 157.0$ & $775.0 \pm 183.0$ & 0.928 \\
\hline Length of hospital stay (day) & $17.7 \pm 6.7$ & $13.3 \pm 2.0$ & $22.5 \pm 6.8$ & 0.001 \\
\hline
\end{tabular}

$\mathrm{RSSI}=$ recipient surgical site infection, S.D.=standard deviation, $\mathrm{yr}=\mathrm{year}, \mathrm{kg} / \mathrm{m}^{2}=\mathrm{kilogram}$ per square meter, $\mathrm{cm}=c e n t i m e t e r, \mathrm{~cm}^{2}=s q u a r e$ centimeter, $\min =$ minute, $\mathrm{ml}=$ milliliter

Overall, RSSI was found in 10 cases; thus the incidence of RSSI in this study was $47.6 \%$. One fibular flap was totally lost due to venous thrombosis, following the data above the success rate for VFBG was $95.2 \%$. Types of RSSI, gram stain, culture and empirical antibiotic treatments of RSSI are shown in Table 4.

Cefazolin $1 \mathrm{~g}$ was administered intravenously as antibiotic prophylaxis in 20 patients, with one case receiving clindamycin $600 \mathrm{mg}$ intravenously due to a history of penicillin anaphylaxis. The most common empirical antibiotics treatment for RSSI was cefazolin
$1 \mathrm{~g}$ plus metronidazole $500 \mathrm{mg}$ intravenously (40.0\%), followed by penicillin $G$ sodium 2 million units plus ceftriaxone $1 \mathrm{~g}(30.0 \%)$.

ASA PS class II and oral contamination were found to be significantly associated with increasing RSSI ( $p$-value $=0.004$ and $p$-value $=0.031$, respectively). Patients with RSSI had a significantly longer length of hospital stay compared to those without RSSI ( $p$-value<0.001). No significant correlation between any of the other variables was observed. 
Table 3 Classification of maxillary and mandibular defects $(n=21)$

\begin{tabular}{ll}
\hline Classification of defect & Number $(\%)$ \\
\hline Mandible & \\
LCL & $6(28.6)$ \\
LC & $6(28.6)$ \\
L & $3(14.3)$ \\
H & $3(14.3)$ \\
Maxilla & \\
IIb & $2(9.5)$ \\
IIIC & $1(4.2)$ \\
\hline
\end{tabular}

$\mathrm{LCL}=$ lateral-center-lateral, $\mathrm{LC}=$ lateral-center, $\mathrm{L}=$ lateral, $\mathrm{H}=$ hemimandible, Ilb=low maxillary defect, not involving floor of orbit and cross midline, IIIc=high maxillary defect, involving floor of orbit, nearly total maxillectomy

Table 4 Recipient surgical site infection data and empirical antibiotic treatment

\begin{tabular}{ll}
\hline Variable & Number (\%) \\
\hline Type of SSI ( $\mathrm{n}=10)$ & \\
Superficial incisional & $2(20.0)$ \\
Space/organ & $5(50.0)$ \\
Deep incisional & $3(30.0)$ \\
Gram stain $(\mathrm{n}=18)$ & \\
Gram-positive cocci & $12(66.7)$ \\
Gram-positive bacilli & $1(5.5)$ \\
Gram-negative cocci & $1(5.5)$ \\
Gram-negative bacilli & $4(22.3)$ \\
Cultures results (n=14) & \\
Streptococcus sp. & $6(42.9)$ \\
K. pneumoniae & $2(14.3)$ \\
Enterobactor sp. & $1(7.1)$ \\
Anaerobic gram-positive cocci & $3(21.5)$ \\
Anaerobic gram-negative bacilli & $1(7.1)$ \\
No growth & $1(7.1)$ \\
Empirical antibiotics (n=10) & \\
Cefazolin+Metronidazole & $4(40.0)$ \\
Penicillin G Sodium+Ceftriaxone & $3(30.0)$ \\
Cefazolin+Clindamycin & $1(10.0)$ \\
Clindamycin+Gentamicin & $1(10.0)$ \\
Penicillin G Sodium & $1(10.0)$ \\
\hline
\end{tabular}

SSI=surgical site infection, K. pneumoniae=Klebsiella pneumoniae, sp. $=$ species

\section{Discussion}

SSI are frequent postoperative complications, especially in head and neck cancer surgery with microvascular free flap reconstruction. ${ }^{4,5}$ Most studies reported incidences of SSI in head and neck cancer surgery ranging from $14.0-50.0 \%$, with various risk factors depending on the study setting. ${ }^{4-8,11,17-21}$ Those studies mentioned SSI in the context of overall head and neck cancer surgery, which used various techniques of soft and hard tissue reconstruction. Only one study reported the incidence of SSI (39.2\%) in patients receiving bony free flap reconstruction to the head and neck, including the fibula, iliac and scapula. ${ }^{9}$ Almost all previous reports included both recipient and donor site infections, for determining the incidence of SSI. In fact, the recipient site (head and neck) surgery, which is a class II (clean-contaminated) wound, has more risk of infection than the donor site surgery, which is a class I (clean) wound. ${ }^{16}$ This retrospective study is the first study, to our knowledge, to report the incidence of SSI specific to the recipient site after oral and maxillofacial reconstruction with VFBG. Additionally, this present study also revealed that: oral contamination was a significant risk factor for RSSI, which is consistent with other studies. ${ }^{4,5}$ Although, the incidence of RSSI in this study is within the range of previous reports ${ }^{4-8,11,17-21}$, it seems to be high compared to most of these studies. This may be because microvascular reconstruction with VFBG is invasive, and entails a high risk of postoperative infection; especially in oral and maxillofacial surgery. These operations often create oral-cervical connected wounds and can expose the VFBG as well as cervical tissue to saliva, and the alimentary-tract or respiratory tract secretions. ${ }^{7}$ Postoperatively, respiration, swallowing and coughing can result in wound dehiscence. ${ }^{9}$ This allows oral microorganism to form infections in the recipients surgical wound in addition to the development of RSSI. We advise meticulous saline irrigation before closing the wound with a watertight seal, in order to reduce the risk of RSSI. 
In this present study, ASA PS class II was also a risk factor for RSSI. However, no significant relationship between RSSI and the presence of systemic diseases, smoking status, alcohol consumption, and BMI was observed. Therefore, ASA PS score may be a good index for a comprehensive health assessment before VFBG. ${ }^{7}$

Although, the incidence of RSSI in this study was high, the failure rate of VFBG was very low. This is probably because of early diagnosis and treatment of RSSI in our hospital, and the high tolerance against infection of the vascularized flap. VFBG is a free tissue transfer with its own blood supply; hence, the immune cells and antibiotics are able to be distributed within and around the VFBG, this is turn helps prevent the spread of causative microorganisms.

This study used cefazolin $1 \mathrm{~g}$ intravenously as a routine antibiotic prophylaxis and clindamycin $600 \mathrm{mg}$ intravenously, for patients who had a history of penicillin allergies. Murphy et al. ${ }^{9}$ found that the rate of RSSI with cefazolin used as an antibiotic prophylaxis for cleancontaminated head and neck osteomyocutaneous free flap was $25.0 \%$; additionally there was no statistical difference observed between the number of SSI patients and non SSI patients. However, previous studies showed that the single use of cefazolin as an antibiotic prophylaxis in major head and neck surgery had a significantly higher rate of wound infections compared to the anaerobic coverage of antibiotics. ${ }^{22,23}$ Veve et al. ${ }^{24}$ and Chiesa-Estomba et al. ${ }^{25}$ suggested that the appropriate antibiotics for prophylaxis in clean-contaminated head and neck surgery; especially for those combining free flap reconstruction, should cover gram-positive, enteric gram-negative and anaerobic bacteria. The recommended antibiotics, for Asian patients, are cefazolin $2 \mathrm{~g}$ in combination with $500 \mathrm{mg}$ of metronidazole and $1.2 \mathrm{~g}$ amoxycillin/clavulanate. For patients with a history of anaphylaxis penicillin allergies, clindamycin alone is not recommended as an antibiotic prophylaxis. Yang et al. ${ }^{6}$ investigated intraoral flora and SSI among high-risk head and neck cancer patients, who underwent resection and free flap reconstruction, and found rapid and significant increases in bacterial resistance to clindamycin. Additionally, some studies revealed that clindamycin prophylaxis alone was a risk factor for SSI in head and neck surgery. ${ }^{9,10}$ This was probably because clindamycin did not cover gramnegative bacteria in these wounds. Therefore, clindamycin should be combined with a secondary drug, so as to cover gram-negative bacteria, or for the consideration of switching to a different antibiotic, such as vancomycin or clarithromycin. $^{25}$

This study used dexamethasone as a perioperative steroid in a number of patients, but such use was not related to RSSI in this study. Howbeit, Kainulainen et al. ${ }^{26}$ showed not only did the use of dexamethasone in oral cancer surgery with microvascular reconstruction not provide any benefits, it also entailed more complications; especially infections, occurring in patients within the control group. Due to the above factors, we suggest that perioperative steroids should not be used routinely. Previous studies also showed that preoperative radiotherapy was not only a risk factor for free flap failure ${ }^{27}$, but that it was also a risk factor of SSI. ${ }^{5,28}$ In our study, all three patients with a history of preoperative radiotherapy developed $\mathrm{RSSI}$, but statistical analysis did not reveal that preoperative radiotherapy was a risk factor of RSSI. This result is in contrast to previous studies ${ }^{5,28}$, this may have been due to the limited sample size of this study.

In our study, patients with RSSI were found to have a significantly higher length of hospital stay than the non-RSSI group, which is consistent with previous studies. ${ }^{11,19}$ Prolonged length of hospital stay in this study may be attributed to most of our RSSI cases requiring extended use of antibiotics, daily irrigation and caring of the infected wound until it improved, as well as physiotherapy at the donor site. Since, prolonged hospitalization has been 
shown to be related to an increased risk for hospitalacquired infections, and deep vein thrombosis as well as incurring higher costs ${ }^{29}$, prevention of RSSI is important for reducing the length of hospitalization.

The limitation of this study was its small sample size, which may have limited the statistical significance of some variables. Therefore, studies with larger sample sizes should be conducted, for further investigation in this field.

\section{Conclusion}

The incidence of recipient site infections after oral and maxillofacial reconstruction with VFBG was high. ASA PS class II, and oral contamination are important risk factors for RSSI.

\section{Conflict of interest}

The authors declare that there are no conflict of interest regarding the publication of this paper.

\section{References}

1. O'Leary MJ, Martin PJ, Hayden RE. The neurocutaneous free fibula flap in mandibular reconstruction. Otolaryngol Clin North Am 1994;27:1081-96.

2. Urken ML. Composite free flaps in oromandibular reconstruction. Arch Otolaryngol Head Neck Surg 1991;117:724-32.

3. Urken ML, Weinberg H, Buchbinder D, Moscoso JF, Lawson W, Catalano PJ, et al. Microvascular free flaps in head and neck reconstruction. report of 200 cases and review of complications. Arch Otolaryngol Head Neck Surg 1994;120: 633-40.

4. Ogihara H, Takeuchi K, Majima Y. Risk factors of postoperative infection in head and neck surgery. Auris Nasus Larynx 2009; 36:457-60.

5. Lee DH, Kim SY, Nam SY, Choi SH, Choi JW, Roh JL. Risk factors of surgical site infection in patients undergoing major oncological surgery for head and neck cancer. Oral Oncol 2011;47:528-31.

6. Yang $\mathrm{CH}$, Chew $\mathrm{KY}$, Solomkin JS, Lin PY, Chiang YC, Kuo YR. Surgical site infections among high-risk patients in cleancontaminated head and neck reconstructive surgery: concor- dance with preoperative oral flora. Ann Plast Surg 2013; 71(Suppl 1):S55-60.

7. Karakida K, Aoki T, Ota Y, Yamazaki H, Otsuru M, Takahashi $M$, et al. Analysis of risk factors for surgical-site infections in 276 oral cancer surgeries with microvascular free-flap reconstructions at a single university hospital. J Infect Chemother 2010;16:334-9.

8. Eder-Czembirek C, Czembirek C, Braun P, Perisanidis C, Seemann R. Surgical site infection in clean-contaminated wounds after multimodal treatment of advanced oral squamous cell carcinoma. J Craniomaxillofac Surg 2016;44:1957-62.

9. Murphy J, Isaiah A, Dyalram D, Lubek JE. Surgical site infections in patients receiving osteomyocutaneous free faps to the head and neck. Does choice of antibiotic prophylaxis matter? J Oral Maxillofac Surg 2017;75:2223-9.

10. Cannon RB, Houlton JJ, Mendez E, Futran ND. Methods to reduce postoperative surgical site infections after head and neck oncology surgery. Lancet Oncol 2017;18:e405-13.

11. Candau-Alvarez A, Linares-Sicilia MJ, Dean-Ferrer A, PérezNavero JL. Role of culture of postoperative drainage fluid in the prediction of infection of the surgical site after major oncological operations of the head and neck. $\mathrm{Br} \mathrm{J}$ Oral Maxillofac Surg 2015;53:200-3.

12. Wilson D, Wakefield M, Owen N, Roberts L. Characteristics of heavy smokers. Prev Med 1992;21:311-9.

13. CDC.gov. Alcohol use and your health [homepage on the Internet]. Atlanta: Centers for Disease Control and Prevention [cited 2020 Mar 10]. Available from: https://www.cdc.gov/ alcohol/fact-sheets/alcohol-use.htm

14. Jewer DD, Boyd JB, Manktelow RT, Zuker RM, Rosen IB, Gullane PJ, et al. Orofacial and mandibular reconstruction with the iliac crest free flap: a review of 60 cases and a new method of classification. Plast Reconstr Surg 1989;84:391403.

15. Brown JS, Rogers SN, McNally DN, Boyle M. A modified classification for the maxillectomy defect. Head Neck 2000; 22:17-26.

16. Mangram AJ, Horan TC, Pearson ML, Silver LC, Jarvis WR. Guideline for prevention of surgical site infection, 1999. Hospital Infection Control Practices Advisory Committee. Infect Control Hosp Epidemiol 1999;20:250-78.

17. Penel N, Fournier C, Lefebvre D, Lefebvre JL. Multivariate analysis of risk factors for wound infection in head and 
neck squamous cell carcinoma surgery with opening of mucosa. Study of 260 surgical procedures. Oral Oncol 2005; 41:294-303.

18. Lotfi CJ, Cavalcanti Rde C, Costa e Silva AM, Latorre Mdo R, Ribeiro Kde C, Carvalho AL, et al. Risk factors for surgical-site infections in head and neck cancer surgery. Otolaryngol Head Neck Surg 2008;138:74-80.

19. Penel N, Mallet $Y$, Roussel-Delvallez M, Lefebvre JL, Yazdanpanah $\mathrm{Y}$. Factors determining length of the postoperative hospital stay after major head and neck cancer surgery. Oral Oncol 2008;44:555-62.

20. Liu SA, Wong YK, Poon CK, Wang CC, Wang CP, Tung KC. Risk factors for wound infection after surgery in primary oral cavity cancer patients. Laryngoscope 2007;117:166-71.

21. Lahtinen S, Koivunen P, Ala-Kokko T, Kaarela O, Ohtonen P, Laurila P, Liisanantti JH. Complications and outcome after free flap surgery for cancer of the head and neck. $\mathrm{Br} J$ Oral Maxillofac Surg 2018;56:684-91.

22. Robbins KT, Byers RM, Cole R, Fainstein V, Guillamondegui OM, Schantz SP, et al. Wound prophylaxis with metronidazole in head and neck surgical oncology. Laryngoscope 1988;98: 803-6.

23. Johnson JT, Yu VL. Antibiotic use during major head and neck surgery. Ann Surg 1988;207:108-11.
24. Veve MP, Davis SL, Williams AM, McKinnon JE, Ghanem TA. Considerations for antibiotic prophylaxis in head and neck cancer surgery. Oral Oncol 2017;74:181-7.

25. Chiesa-Estomba CM, Lechien JR, Fakhry N, Melkane A, Calvo-Henriquez C, de Siati D, et al. Systematic review of international guidelines for perioperative antibiotic prophylaxis in Head \& Neck Surgery. A YO-IFOS Head \& Neck Study Group Position Paper. Head Neck 2019;41:3434-56.

26. Kainulainen S, Törnwall J, Koivusalo AM, Suominen AL, Lassus $P$. Dexamethasone in head and neck cancer patients with microvascular reconstruction: no benefit, more complications. Oral Oncol 2017;65:45-50.

27. Zhou W, Zhang WB, Yu Y, Wang Y, Mao C, Guo CB, et al. Risk factors for free flap failure: a retrospective analysis of 881 free flaps for head and neck defect reconstruction. Int J Oral Maxillofac Surg 2017;46:941-5.

28. Benatar MJ, Dassonville O, Chamorey E, Poissonnet G, Ettaiche M, Pierre CS, et al. Impact of preoperative radiotherapy on head and neck free flap reconstruction: a report on 429 cases. J Plast Reconstr Aesthet Surg 2013;66:478-82.

29. Rosman M, Rachminov O, Segal O, Segal G. Prolonged patients' in-hospital waiting period after discharge eligibility is associated with increased risk of infection, morbidity and mortality: a retrospective cohort analysis. BMC Health Serv Res 2015;15:246. 